OPEN ACCESS

International Journal of

Environmental Research and

Public Health

ISSN 1660-4601

www.mdpi.com/journal/ijerph

Article

\title{
Quantifying and Adjusting for Disease Misclassification Due to Loss to Follow-Up in Historical Cohort Mortality Studies
}

\section{Laura L. F. Scott * and George Maldonado}

Division of Environmental Health Sciences, University of Minnesota School of Public Health, Minneapolis, MN 55455, USA; E-Mail: GMPhD@umn.edu

* Author to whom correspondence should be addressed; E-Mail: 1lfscott@umn.edu; Tel.: +1-281-838-4427.

Academic Editors: Igor Burstyn, Gheorghe Luta and Paul B. Tchounwou

Received: 16 March 2015 / Accepted: 8 October 2015 / Published: 15 October 2015

\begin{abstract}
The purpose of this analysis was to quantify and adjust for disease misclassification from loss to follow-up in a historical cohort mortality study of workers where exposure was categorized as a multi-level variable. Disease classification parameters were defined using 2008 mortality data for the New Zealand population and the proportions of known deaths observed for the cohort. The probability distributions for each classification parameter were constructed to account for potential differences in mortality due to exposure status, gender, and ethnicity. Probabilistic uncertainty analysis (bias analysis), which uses Monte Carlo techniques, was then used to sample each parameter distribution 50,000 times, calculating adjusted odds ratios $\left(O R_{D M-L T F}\right)$ that compared the mortality of workers with the highest cumulative exposure to those that were considered never-exposed. The geometric mean $O R_{D M-L T F}$ ranged between 1.65 (certainty interval (CI): 0.50-3.88) and 3.33 (CI: 1.21-10.48), and the geometric mean of the disease-misclassification error factor $\left(\mathcal{E}_{D M-L T F}\right)$, which is the ratio of the observed odds ratio to the adjusted odds ratio, had a range of 0.91 (CI: 0.29-2.52) to 1.85 (CI: 0.78-6.07). Only when workers in the highest exposure category were more likely than those never-exposed to be misclassified as non-cases did the $O R_{D M-L T F}$ frequency distributions shift further away from the null. The application of uncertainty analysis to historical cohort mortality studies with multi-level exposures can provide valuable insight into the magnitude and direction of study error resulting from losses to follow-up.
\end{abstract}


Keywords: probabilistic bias analysis; Monte Carlo; disease misclassification; loss to follow-up; historical cohort mortality

\section{Introduction}

Epidemiologists have several means available with which to evaluate the exposure-disease relationship in occupational settings. One of the most frequently used methods is the historical cohort mortality study [1,2]. In general, this type of study offers several benefits in that it typically requires less time to complete, is inexpensive compared to other types of studies, and is well-suited for evaluating multiple outcomes and occurrences of rare diseases. However, historical cohort studies are also vulnerable to loss to follow-up, with one method of addressing this being to withdraw lost individuals from the analysis at the time of loss $[1,3,4]$.

Detailed methods for conducting bias analysis (probabilistic uncertainty analysis) of epidemiologic studies have been described by a number of researchers and methodologists [5-12]. Historical cohort mortality studies, however, provide distinct challenges for quantifying study error. First, these types of studies commonly use person-time as the denominator of disease frequency measures; yet bias analysis methods for adjusting an estimate with a person-time denominator have not been described in the peer-reviewed literature. Consequently, to conduct a bias analysis of a cohort mortality study one must use a measure which does not rely on person-time (i.e., odds ratio, incidence proportion ratio, etc.). Although using one of these measures would seem to be a simple solution, it introduces another issue unique to historical cohort mortality studies: disease misclassification due to loss to follow-up, which can result from counting those lost to follow-up who have died as alive at the end of a study. Bias analysis methods to account for this type of disease misclassification have not been previously described. Here, we describe such a method and illustrate how it can be applied to a historical cohort mortality study of New Zealand trichlorophenol workers.

\section{Methods}

\subsection{Error Term for Disease Misclassification Due to Losses}

In 2008, Maldonado [12] detailed the mathematical relationship between a causal relative risk, an observed relative risk, and error terms for study bias. We have provided a modification of this relationship (Equation (1)), where $O R_{D M-L T F}$ is the odds ratio adjusted for disease misclassification due to loss to follow-up, $O R_{\text {observed }}$ is the observed crude odds ratio, and $\mathcal{E}_{i}$ are the terms which quantify the systematic error in a study. Because in this manuscript only one error is being evaluated, the denominator has been simplified to $\varepsilon_{D M-L T F}$, the error term for disease misclassification due to loss to follow-up. $\mathcal{E}_{D M-L T F}$ is calculated by taking the ratio of the observed odds ratio to the adjusted odds ratio.

$$
O R_{D M-L T F}=\frac{O R_{\text {observed }}}{\prod_{i=1}^{n} \varepsilon_{i}}=\frac{O R_{\text {observed }}}{\varepsilon_{D M-L T F}}
$$




\subsection{Crude Odds Ratio}

Using the mortality data described by McBride et al. [13], we calculated a crude odds ratio for the association between ischemic heart disease (IHD) mortality and exposure to 2,3,7,8-tetrachlorodibenzo- $p$-dioxin (TCDD). The odds of IHD death for the group with the highest TCDD exposure was $14 / 148=0.0946$, and the odds of IHD death for "never-exposed" workers was $14 / 451=0.0310$, giving an observed odds ratio of 3.05 and a $95 \%$ confidence interval of $1.42-6.54$ (Table 1).

Table 1. Cell counts used to estimate the crude odds ratio and $95 \%$ confidence limits for the association between occupational TCDD exposure and ischemic heart disease using data reported by McBride et al. [13].

\begin{tabular}{lccc}
\hline \multirow{2}{*}{ Outcome } & \multicolumn{3}{c}{ TCDD Exposure } \\
\cline { 2 - 4 } & $\mathbf{2 0 8 5 . 8}$ ppt-mo & $\mathbf{0 - 2 0 8 5 . 7}$ ppt-mo & Never-Exposed \\
\hline IHD Cases & 14 & 47 & 14 \\
Non-cases & 148 & 925 & 451 \\
Alive & 112 & 826 & 414 \\
Deceased $^{\text {a }}$ & 36 & 99 & 37 \\
\hline & a From causes of death other than
\end{tabular}

\subsection{Number of All-Cause Deaths among Losses to Follow-up}

To estimate the number of workers lost that could have died from IHD for each exposure category, we used a multi-step process (Figure 1). First, we defined a probability distribution for the total number of those lost to follow-up that may have died from any cause for all exposure levels combined. A total of 338 individuals ( $\sim 21 \%$ of the cohort) were lost to follow-up in the cohort mortality study published by McBride and colleagues [13]. We assumed that anywhere from zero to 338 individuals might have died from any cause. Therefore, the minimum and maximum of the probability distribution were set to zero and 338, respectively. We specified the peak of this probability distribution by using the proportions of known deaths observed for the cohort. These ranged from $11.0 \%$, the observed proportion of all deaths in the never-exposed category, to $30.9 \%$, the observed proportion of all deaths in the highest exposure category. The peak number of all-cause deaths for this probability distribution was estimated by multiplying each proportion by the total number of individuals lost. More specifically, $30.9 \%$ of 338 provided a peak value of 104 (Scenarios $1-4$ in Table 2 ) and $11.0 \%$ of 338 provided a peak value of 37 (Scenarios 5-8 in Table 2). We chose a negative binomial distribution-a discrete distribution with more flexibility than the Poisson distribution for providing the desired shape of the probability distribution - with lower and upper truncation points of 0 and 338, respectively, to describe the spread of the number of all-cause deaths in those workers that were lost. These minimum, maximum and peak values determined the probability and shape input of all-cause deaths for each bias-analysis scenario. 
Table 2. Bias-analysis scenarios: description of probability distributions for classification parameters used to estimate the number of workers lost to follow-up that could have died from IHD and corresponding geometric mean errors $\left(\mathcal{E}_{D M-L T F}\right)$, adjusted odds ratios $($ OR bias-analysis certainty intervals.

\begin{tabular}{|c|c|c|c|c|c|c|c|c|c|}
\hline \multirow[b]{2}{*}{ Scenario } & \multirow{2}{*}{$\begin{array}{l}\text { Total All-Cause Deaths } \\
\text { Distribution (Parameters) }\end{array}$} & \multirow[t]{2}{*}{ Total IHD Deaths } & \multicolumn{3}{|c|}{ IHD Deaths by Exposure Status } & \multirow{2}{*}{\multicolumn{2}{|c|}{$\frac{\varepsilon_{D M-L T F}}{95 \% \text { Certainty }}$}} & \multirow{2}{*}{\multicolumn{2}{|c|}{$\begin{array}{c}O R_{D M-L T F} \\
95 \% \text { Certainty } \\
\text { Interval }\end{array}$}} \\
\hline & & & $\begin{array}{c}\text { Direction of } \\
\text { Misclassification }\end{array}$ & $\begin{array}{c}\text { Distribution (Parameters)- } \\
\text { Never-exposed }\end{array}$ & $\begin{array}{l}\text { Distribution (Parameters)- } \\
\geq \mathbf{2 0 8 5 . 8} \text { ppt TCDD-mo }\end{array}$ & & & & \\
\hline 1 & Negative Binomial a $(0.02,3)$ & $\operatorname{BetaPERT}^{\mathbf{b}}\left(0,0.204^{\mathbf{c}} \times \mathrm{AD}, \mathrm{AD}\right)$ & Differential A ${ }^{\mathbf{d}}$ & BetaPERT $(0,3 / 4$ × ID, ID) & $\operatorname{BetaPERT}(0,1 / 2 \times \operatorname{IDE}, \text { IDE })^{\mathbf{e}}$ & 1.85 & $0.78-6.07$ & 1.65 & $0.50-3.88$ \\
\hline 3 & Negative Binomial a $(0.02,3)$ & BetaPERT $\left(0,0.139^{\mathrm{c}} \times \mathrm{AD}, \mathrm{AD}\right)$ & Differential A & BetaPERT $(0,3 / 4 \times$ ID, ID) & BetaPERT $(0,1 / 2 \times$ IDE, IDE) & 1.62 & $0.74-4.93$ & 1.88 & $0.62-4.11$ \\
\hline 5 & Negative Binomial ${ }^{\mathbf{f}}(0.027,2)$ & $\operatorname{BetaPERT}(0,0.204 \times \mathrm{AD}, \mathrm{AD})$ & Differential A & BetaPERT $(0,3 / 4 \times$ ID, ID) & $\operatorname{BetaPERT}(0,1 / 2 \times$ IDE, IDE $)$ & 1.50 & $0.87-3.92$ & 2.03 & $0.78-3.51$ \\
\hline 7 & Negative Binomial ${ }^{\mathbf{f}}(0.027,2)$ & BetaPERT $(0,0.139 \times \mathrm{AD}, \mathrm{AD})$ & Differential A & BetaPERT $(0,3 / 4 \times$ ID, ID) & BetaPERT $(0,1 / 2 \times$ IDE, IDE) & 1.43 & $0.87-3.65$ & 2.13 & $0.83-3.50$ \\
\hline 2 & Negative Binomial a $(0.02,3)$ & BetaPERT $(0,0.204 \times \mathrm{AD}, \mathrm{AD})$ & Differential B $\mathbf{g}$ & BetaPERT $(0,1 / 4$ × ID, ID) & BetaPERT $(0,1 / 2 \times$ IDE, IDE) & 0.91 & $0.29-2.52$ & 3.33 & $1.21-10.48$ \\
\hline 4 & Negative Binomial a $(0.02,3)$ & BetaPERT $(0,0.139 \times \mathrm{AD}, \mathrm{AD})$ & Differential B & BetaPERT $(0,1 / 4$ × ID, ID) & BetaPERT $(0,1 / 2 \times$ IDE, IDE) & 0.92 & $0.32-2.37$ & 3.31 & $1.29-9.65$ \\
\hline 6 & Negative Binomial ${ }^{\mathbf{f}}(0.027,2)$ & $\operatorname{BetaPERT}(0,0.204 \times \mathrm{AD}, \mathrm{AD})$ & Differential B & BetaPERT $(0,1 / 4 \times$ ID, ID) & BetaPERT $(0,1 / 2 \times$ IDE, IDE) & 0.95 & $0.42-1.98$ & 3.20 & $1.54-7.26$ \\
\hline 8 & Negative Binomial $^{\mathbf{f}}(0.027,2)$ & BetaPERT $(0,0.139 \times \mathrm{AD}, \mathrm{AD})$ & Differential B & $\operatorname{BetaPERT}(0,1 / 4 \times$ ID, ID) & BetaPERT $(0,1 / 2 \times$ IDE, IDE) & 0.96 & $0.46-1.86$ & 3.18 & $1.64-6.64$ \\
\hline
\end{tabular}

$\mathrm{AD}$, number of total all-cause deaths; ID, number of total IHD deaths; IDE, number of IHD deaths for those workers ever-exposed; ${ }^{\mathrm{a}}$ Negative binomial distribution (probability, shape) - probability and shape were determined based on minimum, likeliest and maximum counts of $(0,104,338)$; ${ }^{\mathbf{b}}$ BetaPERT distribution (minimum, likeliest, maximum); ${ }^{\mathrm{c}}$ 0.204: proportion of all-cause deaths due to IHD among non-Maori males; 0.139: proportion of all-cause deaths due to IHD among Maori females; ${ }^{\mathbf{d}}$ Never-exposed more likely to be misclassified as alive than highest exposed; ${ }^{\mathbf{e}}$ The maximum value for this distribution is capped at 112 , which is the number of individuals in the highest exposure group (i.e., $\geq 2085.8$ ppt-mo) that were classified as living non-cases; ${ }^{\mathrm{f}}$ Negative binomial distribution (probability, shape) - probability and shape were determined based on minimum, likeliest and maximum counts of $(0,37,338) ;{ }^{\mathrm{g}}$ Never-exposed less likely to be misclassified as alive than highest exposed. 


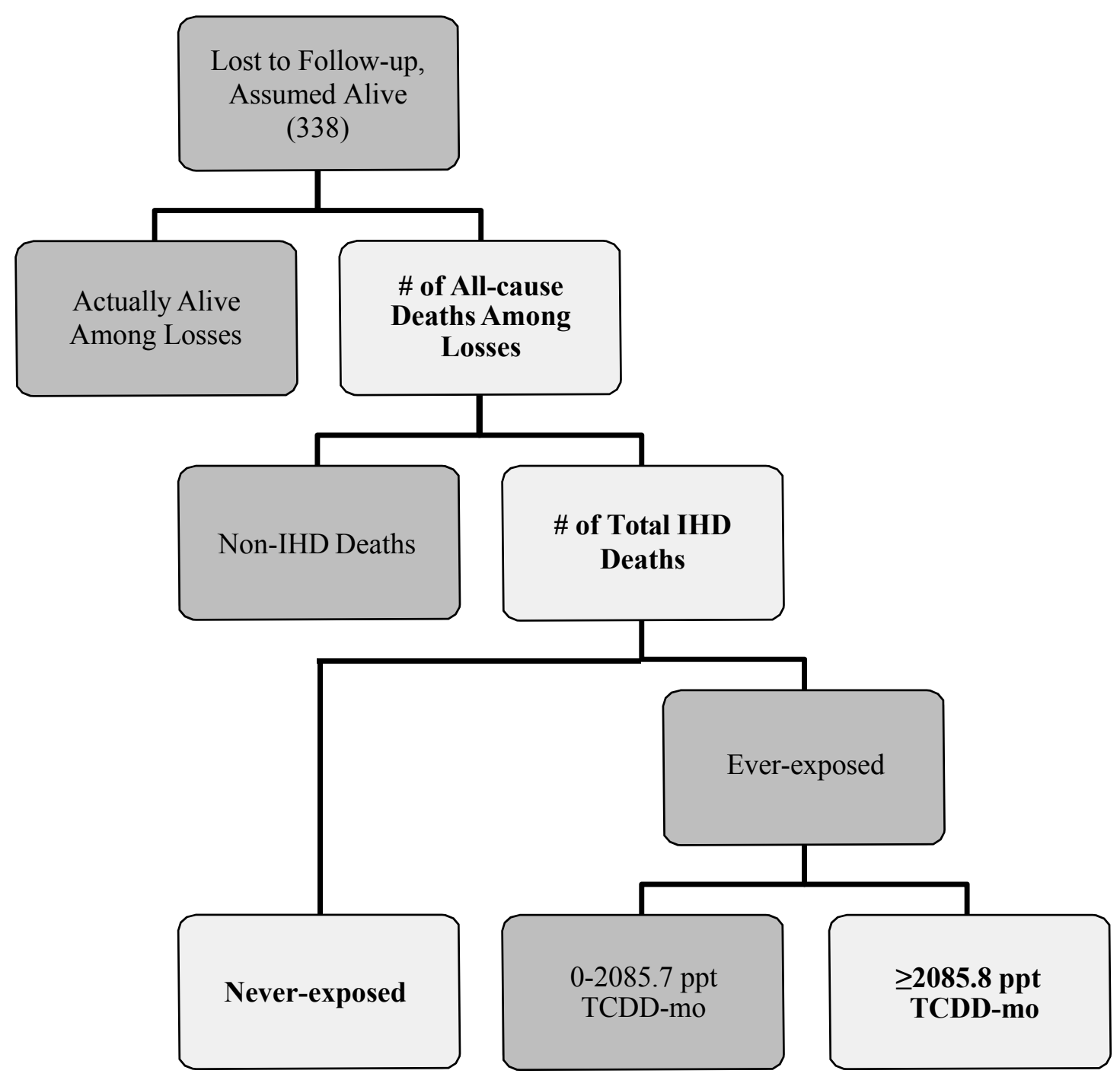

Figure 1. Flow diagram describing how losses to follow-up in Mcbride et al. [13] could result in outcome misclassification. Lighter shapes with bolded text indicate the parameters that were specified in our bias analysis.

\subsection{Number of Total IHD Deaths among Losses to Follow-Up}

Next, we used 2008 mortality data for the New Zealand population [14] to estimate the total number of deaths from IHD for all exposure levels combined. The proportion of New Zealanders who died from IHD varied by both gender and ethnicity, with the proportion of IHD deaths the highest in non-Maori males (20.4\%) and the lowest in Maori females (13.9\%). The BetaPERT (i.e., PERT) distribution, which is derived from the beta distribution and is a smoother alternative to the triangular distribution, was specified as the probability distribution for this disease-classification parameter. We selected this distribution over the negative binomial and Poisson distributions because (1) it is considered to be ideal for modeling expert opinion of a variable [15], (2) it was much more flexible than the Poisson distribution, and (3) the maximum and likeliest values of the distribution, which were dependent on the total number of all-cause deaths selected in the first step, could easily be varied. For example, if the number of all-cause deaths was 100 for a bias-analysis simulation trial, then the distribution for the number of deaths from IHD would range from 0 to 100 with a likeliest value of 13.9, 
assuming $13.9 \%$ of all-cause deaths were due to IHD. Since the BetaPERT distribution is continuous and we are interested in estimating discrete counts, we used the TRUNC function in Excel to remove the decimal portion of each bias-analysis simulation trial value. The difference in the adjusted odds ratios and error terms estimated with and without use of the TRUNC function was negligible.

\subsection{Number of IHD Deaths among Losses to Follow-Up: Never-Exposed}

For the next step, we specified a probability distribution for the number of IHD deaths for the "never-exposed" group. The BetaPERT distribution, along with the TRUNC function in Excel, was also used for this parameter. The maximum and likeliest values were adjusted in a similar manner to that for the total number of IHD deaths. The maximum was set to equal the total number of IHD deaths selected in the previous bias-analysis simulation step. Since the total number that died of IHD could be categorized into one of three exposure groups (i.e., "never-exposed", "0-2085.7 ppt-mo", " $\geq 2085.8$ ppt-mo"), we used simple fractions to determine the likeliest value of this probability distribution depending on whether IHD deaths among the "never-exposed" were (1) more likely to be misclassified as those in the highest exposure group (Differential A) or (2) less likely to be misclassified as those in the highest exposure group (Differential B). When it was assumed that the "never-exposed" were more likely to be misclassified compared to those in the highest exposure group, the likeliest value was set to equal 3/4 the total number of IHD deaths. Under the second assumption, the likeliest value was set to equal 1/4 the total number of IHD deaths. For example, if the total number of IHD deaths selected in the second step of a bias-analysis simulation trial is 24 , then the likeliest values for the "never-exposed" group would be 18 and six, respectively.

\subsection{Number of IHD Deaths among Losses to Follow-Up: $\geq 2085.8$ ppt TCDD-mo}

Last, a probability distribution for the number of IHD deaths among workers with the highest exposure (i.e., $\geq 2085.8$ ppt TCDD-mo) was specified. For this parameter, we again chose to use the BetaPERT distribution, truncating each trial value at the decimal point to obtain a whole number. Using the IF function in Excel, the distribution maximum was set to equal the number of IHD deaths for those "ever-exposed" up to 112, which is the number of individuals in the highest exposure group that were classified as living non-cases, and equal to 112 when the total number of "ever-exposed" that were potentially misclassified as alive (i.e., died of IHD) was greater than 112 . The likeliest value of the probability distribution for the highest exposure group was set to equal $1 / 2$ the number of IHD deaths for the "ever-exposed" workers so that approximately $3 / 8($ i.e., $3 / 4 \times 1 / 2)$ and $1 / 8($ i.e., $1 / 4 \times 1 / 2)$ of the total number of IHD deaths would fall into the highest exposure category.

For example, the probability distribution input used for Scenario 1 is shown in Figure 2. In this scenario, the distribution for the total number of all-cause deaths among those lost to follow-up ranges from 0 to 338 and peaks at 104 (30.9\% of the number lost to follow-up). Assuming 104 all-cause deaths, the distribution for the total number of IHD deaths would then range from 0 to 104, with the highest probability at 21.2 or $20.4 \%$ of 104 . Given 21 total IHD deaths, the likeliest number of workers misclassified as alive would then be 15 for the "never-exposed" category $(3 / 4 \times \#$ IHD deaths $=3 / 4 \times 21$ $\approx 15$ ). For the highest exposure group, the likeliest number of workers lost to follow-up that may 
have died from IHD would be three ((\# total IHD deaths $-\#$ never-exposed IHD deaths $) \times 1 / 2=(21-15) \times$ $1 / 2=3)$.
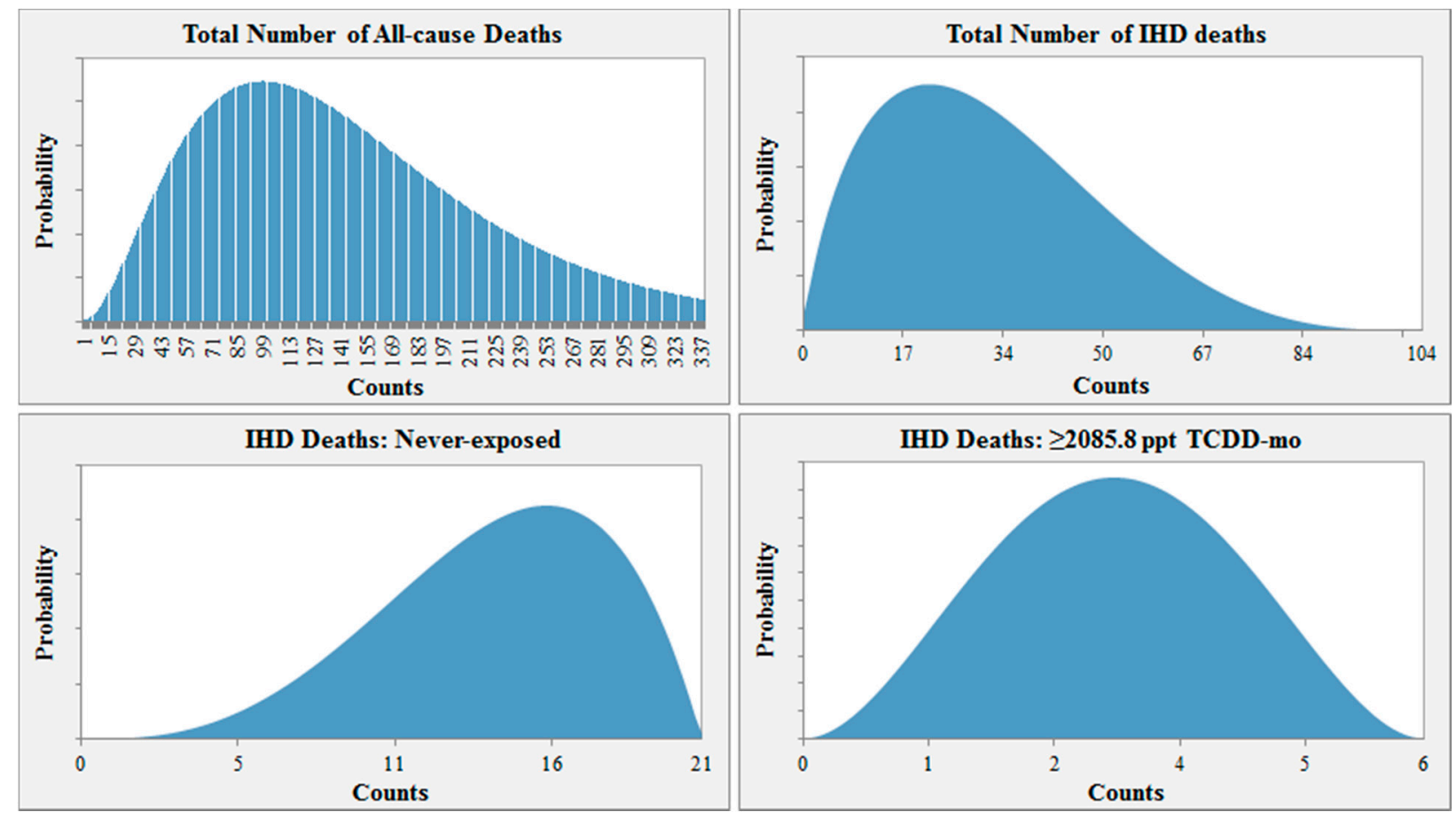

Figure 2. Example of parameter distribution input for Scenario 1.

\subsection{Scenarios and Monte Carlo Simulation Methods}

Combining the different distributions for each classification parameter resulted in eight scenarios (Table 2). The distributions of the classification parameters were sampled with Crystal Ball software [16] to generate adjusted counts of IHD cases and non-cases by exposure group, which we used to calculate adjusted odds ratios and study error. Since Crystal Ball will not allow the program to proceed when zero (or a number truncated to zero) is selected for a given classification parameter, we created a conditional action-using the IF function in Excel-whereby the program would automatically set the ensuing classification parameter values to zero anytime this occurred. For each of the eight scenarios, we conducted 50,000 trials to generate frequency distributions for $O R_{D M-L T F}$ and $\mathcal{E}_{D M-}$ ${ }_{L T F}$ as well as $95 \%$ certainty intervals. Under specific conditions, a $95 \%$ certainty interval may approximate a $95 \%$ Bayesian posterior probability interval, such that there is a $95 \%$ chance that the true estimate for the sample population will fall within the interval [17-19]. This interpretation is different from that of a $95 \%$ confidence interval, which is defined as a range of values that will include the true parameter value $95 \%$ of the time.

\section{Results}

Results for each simulation of the probabilistic uncertainty analysis are summarized in Table 2 and Figure 3. The geometric mean of the error term for disease misclassification due to loss to follow-up ( $\left.\mathcal{E}_{D M-L T F}\right)$ had a range of 0.91 to 1.85 . The geometric mean adjusted odds ratio $\left(O R_{D M-L T F}\right)$ ranged between 1.65 and 3.33. Estimated certainty intervals $(\mathrm{CI})$ for the geometric mean $O R_{D M-L T F}$ excluded the null for 
all four scenarios in which those categorized as "never-exposed" were less likely to be misclassified as alive than workers in the highest exposure category.

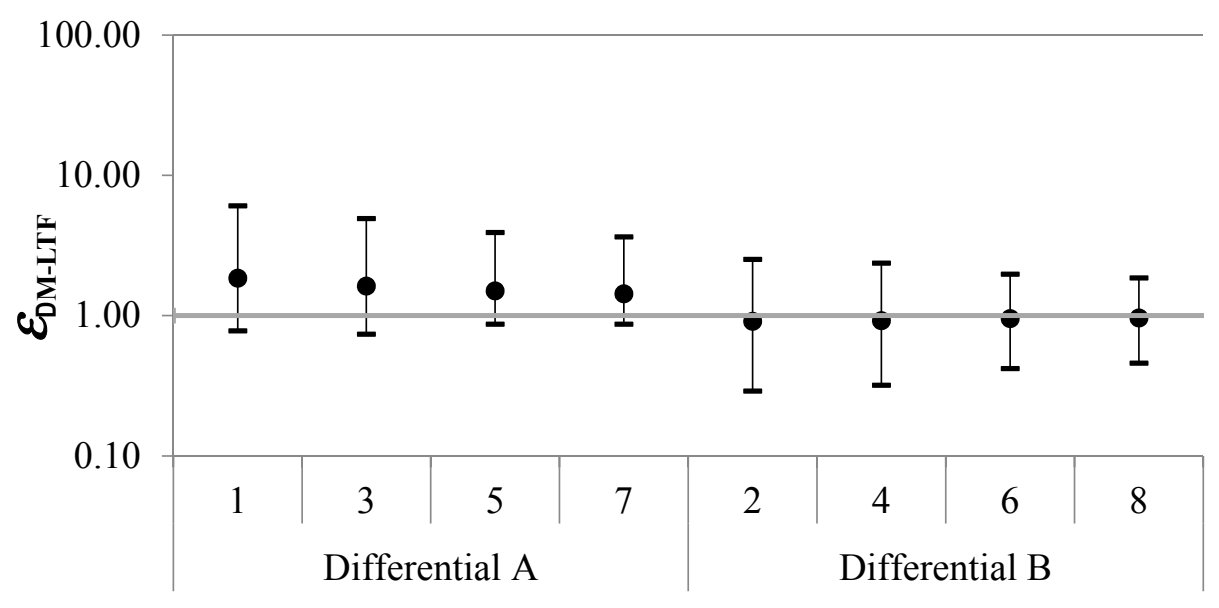

Scenario

(a)

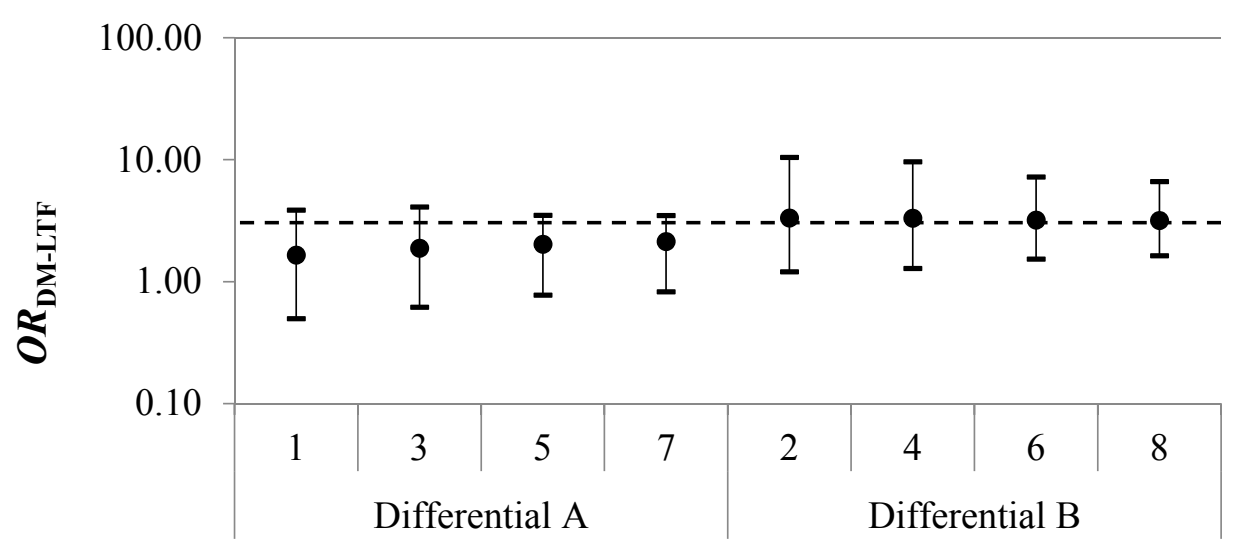

Scenario

(b)

Figure 3. Geometric mean errors $\left(\mathcal{E}_{D M-L T F}\right)(\mathbf{a})$, adjusted odds ratios $\left(O R_{D M-L T F}\right)(\mathbf{b})$ and $95 \%$ certainty intervals by scenario. The dashed horizontal black line in (b) indicates the crude odds ratio $\left(O R_{\text {observed }}\right)$ of 3.05. In the Differential A scenarios, the "never-exposed" were more likely to be misclassified as alive than the highest exposed. In the Differential B scenarios, the "never-exposed" were less likely to be misclassified as alive than the highest exposed.

The direction of the error was primarily determined by the exposure classification parameters. In the four scenarios where workers with the highest exposure were more likely than those never-exposed to be misclassified as alive, the adjusted OR moved away from the null (i.e., the crude OR was biased toward the null). In contrast, when the never-exposed group had a greater proportion misclassified as non-cases than the highest exposure group (Scenarios 1, 3, 5, and 7), adjustment for study bias due to loss to follow-up resulted in a shift of the $O R_{D M-L T F}$ frequency distributions toward the null, lessening the observed effect for the exposure-disease relationship (Figure 4). 
In all the scenarios we examined, uncertainty about the amount of disease misclassification due to assuming that lost subjects were alive resulted in uncertainty about the magnitude of the TCDD-IHD association estimate.

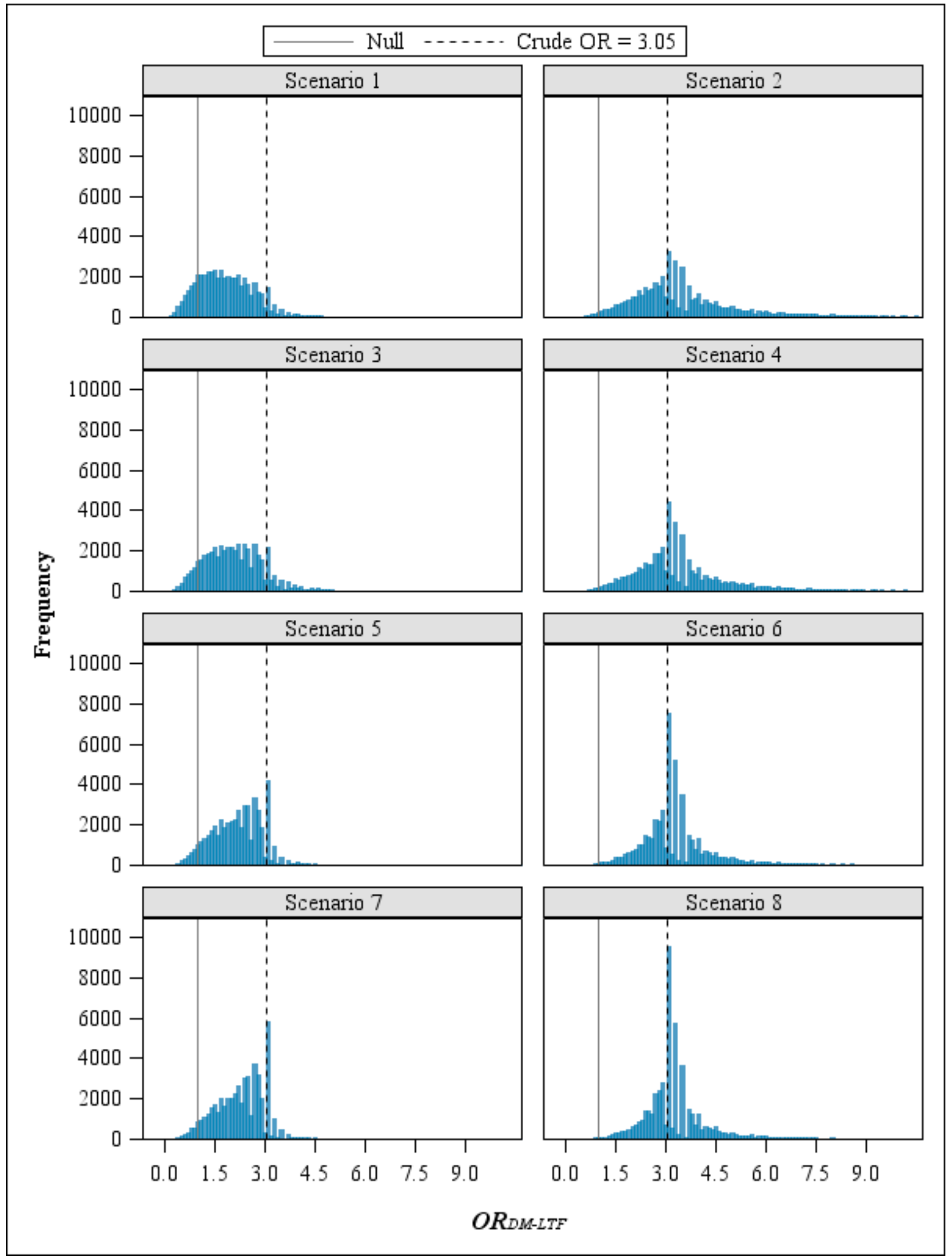

Figure 4. Frequency distributions of $O R_{D M-L T F}$ by scenario. 


\section{Discussion}

Here we demonstrated that losses to follow-up in a historical cohort mortality study can cause disease misclassification. This source of disease misclassification is different from the misclassification that may result from using death certificate or other mortality data and is unique to historical cohort mortality studies. Currently, methods using person-time to conduct probabilistic uncertainty analyses have not been published. As such, one must use either the incidence proportion ratio or the odds ratio as the estimator when conducting bias analyses of these studies. When using either of these measures, however, those lost to follow-up are consequentially counted as alive even though some may have died from the disease of interest. For both measures, study subjects contribute to the denominator but have no opportunity to contribute to the numerator. The only way to prevent this would be to exclude anyone who was lost, but this creates other potential problems and has never been an accepted method for addressing losses to follow-up.

Since we had no reason to assume that disease misclassification caused by losses to follow-up would not affect the association measure for occupational exposure to TCDD and IHD mortality, we developed a probabilistic bias analysis method to adjust for this source of systematic error using Monte Carlo simulations. In developing this method, we encountered several issues that challenged the implementation of our approach. First, very little information was available from the original study [13] on how losses were distributed across the exposure categories. This would likely be easier to address when the exposure variable is dichotomous, but can become particularly complicated with multi-level exposures. We believed that the best method of making the process more manageable was to divide it into multiple steps, which also allowed us to check the accuracy of our process at different points. In addition, it was necessary to calculate the number of living subjects in each exposure group and set this as the maximum distribution value when the total number for a given step exceeded the number of living that could have been misclassified as alive. For example, 148 workers were categorized as non-cases with exposure $\geq 2085.8$ pt TCDD-mo, but only 112 of these employees were classified as alive at the end of the study. The other 36 subjects died of causes other than IHD and could not have been potentially misclassified as alive due to losses to follow-up. Accordingly, we used the IF function in Excel to set the distribution maximum for the number of IHD deaths in the exposure group " $\geq 2085.8$ ppt TCDD-mo" equal to 112 when the total number of "ever-exposed" that were potentially misclassified as alive (i.e., died of IHD) was greater than 112. Otherwise, the maximum value for this parameter equaled the number of IHD deaths among those lost that were "ever-exposed". We also addressed a variety of concerns with regard to the simulation software used and the distributions available. For the number of all-cause deaths among losses, we chose a negative binomial distribution because it was discrete and provided the flexibility we needed to describe the distribution for this parameter. Unfortunately, the negative binomial distribution is determined by probability and shape, which are not easily varied, so it was not an ideal distribution for the remaining three classification parameters - each of which are dependent on the preceding step. We initially considered the Poisson distribution because the peak $(\lambda)$ could be determined based on the trial value of another classification parameter. However, this distribution was not as flexible as we had hoped. For example, if the number of all-cause deaths among losses was 104, the distribution for the total number of IHD deaths would need to peak at 21 , assuming a proportion of $20.4 \%$, and have a range of 0 to 104 . With a 
Poisson distribution, the probability of the simulation choosing a count less than 9 or greater than 37 was zero, effectively excluding the majority of potential values. Rather, we chose to use the continuous BetaPERT distribution - which is renormalized over a finite range other than $(0,1)$, re-parameterized by the minimum, maximum, and mode [15], and very flexible - and truncate the selected trial values to whole numbers. Comparison of the adjusted odds ratios and error terms estimated with and without use of the TRUNC function demonstrated any differences were negligible. Last, it should be noted that the simulation software we used would suspend a run anytime zero was selected as a trial value for the first two parameters or when the total number "ever-exposed" that were potentially misclassified as alive was zero. To address this, we used the IF function in Excel to set the subsequent classification parameter values to zero when any of the described conditions occurred (e.g., if a trial value for the total number of IHD deaths among losses was zero, then all successive parameter values for that trial would be zero). We believe this is a superior alternative to excluding these trials.

In the example described here, we demonstrated that the magnitude of the error from disease misclassification due to loss to follow-up can be substantial. The certainty intervals for $O R_{D M-L T F}$ in the simulations for this bias analysis were quite wide, with the distance between the upper and lower bounds ranging from 2.67 to 9.27. While the exposure parameters ("IHD deaths: never-exposed" and "IHD deaths: $\geq 2085.8$ ppt-mo") were the main determinants of the location of the geometric mean adjusted odds ratios, the width of the certainty intervals was likely influenced more by the degree of misclassification.

By using a probabilistic method that specifies a range of values for the classification parameters under a variety of thoughtfully constructed scenarios, our approach allows one to estimate intervals that may better represent the level of uncertainty from systematic study error in a given exposure-disease relationship. Compared to other techniques such as simple sensitivity analysis and inverse probability weighting (IPW), use of probabilistic bias analysis may be more advantageous because it does not rely on conditional weighting estimates or single sensitivity and specificity values. One of the primary limitations of IPW is that the model estimating the weights is built as a function of subject characteristics (e.g., age, gender). Anything that might be related to follow-up should be part of the model building process. Yet, when characteristics that may be predictive of follow-up are not measured or collected as part of the study, use of this method may actually introduce bias. Our method, however, allows one to use external data to adjust for any characteristic - whether or not measured as part of the initial study - that may affect follow-up or the outcome of interest.

Our primary objectives were to illustrate how to adjust an odds ratio for disease misclassification resulting from losses to follow-up in a historical cohort mortality study and to evaluate the effect of this source of error. It is likely, therefore, that uncertainty about the magnitude of other study limitations; for example, exposure misclassification would further increase the uncertainty about the TCDD-IHD association. Additionally, the method described here is contingent on the distributions constructed for each of the classification parameters, with the usefulness of the results limited by the accuracy of those distributions. For example, we used New Zealand mortality data from 2008, which assumes the proportion of deaths from IHD was constant over the entire study period evaluated by McBride et al. [13]. However, the New Zealand Ministry of Health reported that the percentage of IHD deaths declined over the 1980-2008 time frame [14] and we, therefore, may have underestimated the number lost to follow-up that died from IHD and the level of uncertainty. 


\section{Conclusions}

In summary, our method can be employed to quantify and adjust for disease misclassification due to losses to follow-up in any historical cohort mortality study with a defined polytomous exposure variable. Use of our probabilistic bias method to adjust for this source of systematic error may well be a considerable improvement over the standard conjecture that, most often, incorrectly assumes such error would be non-differential and have little or no effect on the observed exposure-disease relationship.

\section{Acknowledgments}

Support for this research was provided by the National Institute of Occupational Safety and Health (NIOSH) Training Program (Grant \# T42/OH008434) and the University of Minnesota School of Public Health Dean's Scholars Program. The contents of this manuscript are solely the responsibility of the authors and do not necessarily represent the official views of NIOSH.

\section{Author Contributions}

Laura Scott identified the methodological problem addressed in this manuscript. With the assistance of George Maldonado, Laura Scott developed the multi-step, Monte Carlo method described as a solution to this issue. Ms. Scott had primary responsibility for all analyses and preparation of the manuscript, with input from George Maldonado. This manuscript is part of Laura Scott's doctoral research, and George Maldonado is her thesis advisor.

\section{Conflicts of Interest}

The authors declare no conflict of interest.

\section{References}

1. Vena, J.E.; Sultz, H.A.; Carlo, G.L.; Fiedler, R.C.; Barnes, R.E. Sources of bias in retrospective cohort mortality studies: A note on treatment of subjects lost to follow-up. J. Occup. Med. 1987, 29, 256-261.

2. Swaen, G.M.; Meijers, J.M. Influence of design characteristics on the outcome of retrospective cohort studies. Brit. J. Ind. Med. 1988, 45, 624-629.

3. Savitz, D.A.; Moure, R. Treatment of subjects lost to follow-up: Effect on oil refinery cancer risks. J. Occup. Med. 1988, 30, 89-91.

4. Checkoway, H.; Pearce, N.; Kriebel, D. Monographs in epidemiology and biostatistics. In Research Methods in Occupational Epidemiology, 2nd ed.; Oxford University Press: New York, NY, USA, 2004; p. 372.

5. Fox, M.P.; Lash, T.L.; Greenland, S. A method to automate probabilistic sensitivity analyses of misclassified binary variables. Int. J. Epidemiol. 2005, 34, 1370-1376.

6. Jurek, A.M.; Lash, T.L.; Maldonado, G. Specifying exposure classification parameters for sensitivity analysis: Family breast cancer history. Clin. Epidemiol. 2009, 1, 109-117. 
7. Lash, T.L.; Fink, A.K. Semi-automated sensitivity analysis to assess systematic errors in observational data. Epidemiology 2003, 14, 451-458.

8. Lash, T.L.; Fox, M.P.; Fink, A.K. Statistics for biology and health. In Applying Quantitative Bias Analysis to Epidemiologic Data; Springer: Dordrecht, The Netherlands; New York, NY, USA, 2009; p. 192.

9. Jurek, A.M.; Greenland, S. Adjusting for multiple-misclassified variables in a study using birth certificates. Ann. Epidemiol. 2013, 23, 515-520.

10. Jurek, A.M.; Maldonado, G.; Greenland, S. Adjusting for outcome misclassification: The importance of accounting for case-control sampling and other forms of outcome-related selection. Ann. Epidemiol. 2013, 23, 129-135.

11. Jurek, A.M.; Maldonado, G.; Spector, L.G.; Ross, J.A. Periconceptional maternal vitamin supplementation and childhood leukaemia: An uncertainty analysis. J. Epidemiol. Community Health 2009, 63, 168-172.

12. Maldonado, G. Adjusting a relative-risk estimate for study imperfections. J. Epidemiol. Community Health 2008, 62, 655-663.

13. McBride, D.I.; Collins, J.J.; Humphry, N.F.; Herbison, P.; Bodner, K.M.; Aylward, L.L.; Burns, C.J.; Wilken, M. Mortality in workers exposed to 2,3,7,8-tetrachlorodibenzo-p-dioxin at a trichlorophenol plant in New Zealand. J. Occup. Environ. Med. 2009, 51, 1049-1056.

14. Mortality and Demographic Data 2008; New Zealand Ministry of Health: Wellington, New Zealand, 2011.

15. Vose, D. Risk Analysis: A Quantitative Guide, 2nd ed.; Wiley: Chichester, UK; New York, NY, USA, 2000; p. 418.

16. Crystal Ball; Oracle Corporation: Redwood Shores, CA, USA, 2014.

17. Greenland, S. Multiple-bias modelling for analysis of observational data. J. R. Stat. Soc. A 2005, 168, 267-306.

18. Jurek, A.M.; Maldonado, G.; Greenland, S.; Church, T.R. Uncertainty analysis: An example of its application to estimating a survey proportion. J. Epidemiol. Community Health 2007, 61, 650-654.

19. MacLehose, R.F.; Gustafson, P. Is probabilistic bias analysis approximately Bayesian? Epidemiology 2012, 23, 151-158.

(C) 2015 by the authors; licensee MDPI, Basel, Switzerland. This article is an open access article distributed under the terms and conditions of the Creative Commons Attribution license (http://creativecommons.org/licenses/by/4.0/). 